\title{
Optimal sizing of battery energy storage system in microgrid system considering load shedding scheme
}

\author{
Thongchart Kerdphol*, Yaser Qudaih, Yasunori Mitani, Princess Garasi \\ Department of Electrical and Electronics Engineering, Kyushu Institute of Technology, Kitakyushu-shi, Fukuoka, 804-8550, Japan
}

\begin{abstract}
In the past, Battery Energy Storage System (BESS) is used for primary frequency regulation. As developments in batteries progress, advancement in applications of BESS including the implementation in high power penetration is expected. Load shedding is one of frequency control methods during stand-alone operation, and the performance of frequency control improves in combination with BESS. However, without optimal size of BESS, it can cause the oscillations to the system. Thus, this article proposes the feasibility of using optimal BESS with load shedding scheme when the microgrid is disconnected from a main source. In this paper, the analytic algorithm-based DPL (DIgSILENT Programming Language) script is developed and presented to determine the optimal size of BESS with load shedding scheme. Results show that the optimal size of BESS-based analytic algorithm with load shedding scheme can achieve higher performance of frequency control compared to the BESS without optimal size. The optimal sizing method of BESS with load shedding scheme is achieved by using a tool for analysis of industrial, utility and commercial electrical power system called DPL script.
\end{abstract}

Keywords: Battery energy storage system, dynamic system, frequency control, load shedding, optimal sizing method

\section{Introduction}

Rapid decrease of fossil fuel resources, increase of electricity demand and environmental concerns associated with conventional generators call for an amendment of alternative electric energy generation method worldwide. Microgrid system is being considered as one of the solutions to this energy concern, and is gaining more attention recently. A microgrid is a principle concept integrating distributed generation sources (DGs). It can be viewed as a group of distributed generation sources connected to the loads in which the DGs can be fed to the loads alone or be fed to the utility grid [1]. As the output characteristics of these DGs are quite different from the conventional energy sources, the system should be capable of handling unexpected fluctuation and maintaining system reliability. Moreover, a microgrid can operate in either stand-alone or grid-connection mode. It is capable to work in parallel with the utility grid, with the capacity to switch to stand-alone operation in case of emergency situation in the grid. When an islanding operation occurs where a DG or a group of DG sources continue to supply the microgrid system that has been separated from the utility grid, the system needs to have a master generator which can provide voltage and frequency support [2]. Generally, a synchronous generator can fulfil this demand. When there is no synchronous generator, Voltage Source Converter (VSC) interfaced batteries can act as the master control. Thus, storage devices serve as an important aspect in microgrid operations. Presently, battery systems with the capacity of $2 \mathrm{MW}$ have already been utilized to support a microgrid [3].

Battery Energy Storage System (BESS) can be used in various aspects of the power system. BESS is presently used as one key factor for sustainable energy in many countries particularly in Europe, America and Japan. Advantages of BESS include improvement of the system frequency especially when BESS is used for system frequency control. In the case of small disturbance, BESS is discharged when the system frequency is lower than $50 \mathrm{~Hz}$. On the other hand, BESS is charged when the system frequency is higher

\footnotetext{
* Manuscript received May 13, 2013; revised July 24, 2013.

Corresponding author: Thongchart Kerdphol; Tel.: +8-193-884-3243; E-mail address: n589504k@mail.kyutech.jp.

doi: $10.12720 /$ sgce.4.1.22-29
} 
than $50 \mathrm{~Hz}$. In the case of large disturbance, BESS can enhance the performance of the system frequency control by integrating BESS with under frequency load shedding scheme, or under frequency generation trip, and over frequency generation trip. With these different functions, BESS can offer a good solution. It can be concluded that BESS is a rapid and flexible element for the power system [4], [5]. Moreover, the purpose of optimal BESS is to smoothen the power in a system with wind or solar energy. In such a system, BESS can play a role by absorbing the surplus power and compensating the power shortage due to the uncertainties of renewable energy. However, installation of large or inappropriate size of BESS can cause frequency problems to the system and may increase the cost of the system. For these reasons, optimal sizing of BESS is an essential factor for a power system [6-8].

For stand-alone operation, many optimal sizing methods have been already studied including singleobjective [9] and multi-objective approaches [10]. This article is based on multi-objective approach. The objective of this paper is to improve the frequency control and reduce the operating cost of microgrid by integrating load shedding scheme with optimal sizing of BESS. The proposed methodology is based on analytic algorithm in order to find the optimal size of BESS.

This paper can be divided into five parts. The first part is the introduction which discusses the brief introduction of BESS and the optimal sizing methods. Secondly, it describes about the dynamic model of BESS, the model of the microgrid system. The third part discusses the structure of developed program and the optimal sizing method with load shedding scheme. The fourth part reveals the simulation results under the condition of stand-alone operation in the microgrid system. Lastly, the fifth part is the conclusion.

\section{System Configuration}

\subsection{Microgrid system}

A typical characteristic of a microgrid is that it can be operated either in grid-connected operation or stand-alone operation. Under normal operation, the microgrid is connected to the utility grid. Fig. 1 shows the microgrid system which consists of a 1.2 MW mini-hydro generator, $2 \mathrm{MW}$ hydro generator and 3 MW photovoltaic sources. BESS is connected to the microgrid system at bus MSR. The system also consists of group of feeders which could be part of a distribution system. There are some critical loads which require a local generation and the non-critical load feeders are not connected to any local generation.

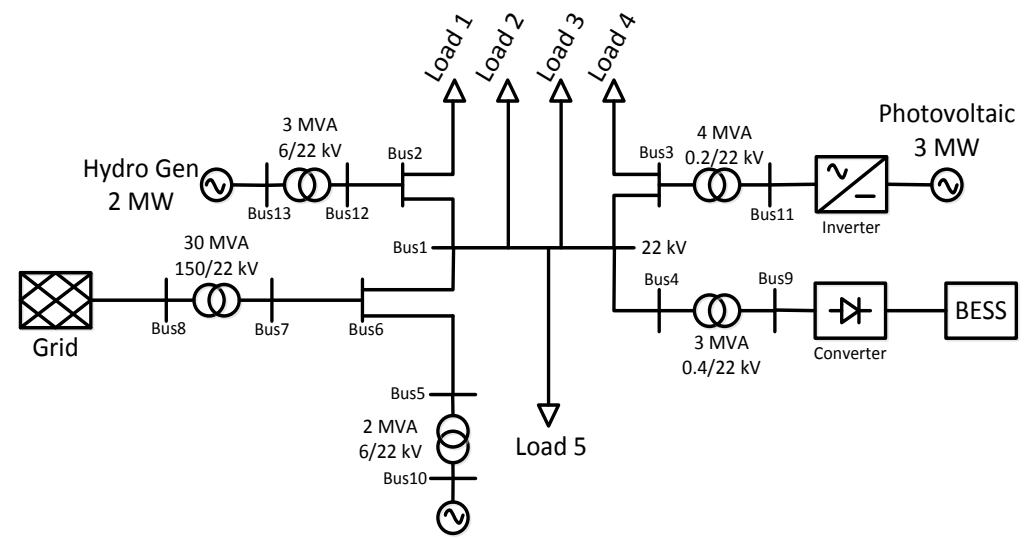

Fig. 1. Microgrid architecture.

\subsection{Battery energy storage system (BESS)}

BESS can be utilized in several implementations such as peak shaving, real power control and load leveling. This paper uses BESS for enhancing the performance of frequency control as BESS can provide active power compensation in a short period of time. This strategy can be considered as a new method. Recently, a huge number of electric companies and independent system operators have shown growing 
interest in BESS due to decreasing cost of batteries. With the fast development of technologies, BESS is expected to be used in several applications including the one proposed in this paper.

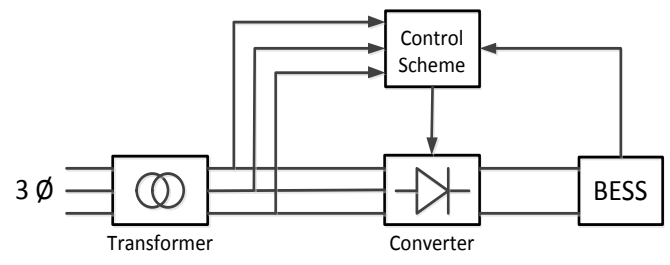

(a)

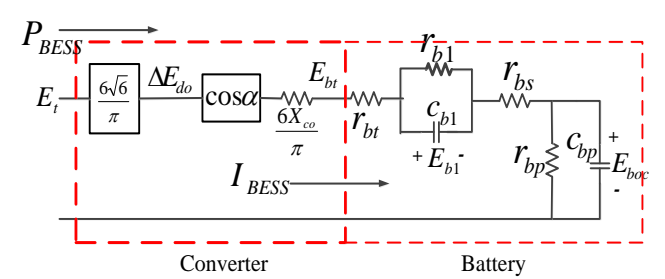

(b)

Fig. 2. BESS structure: (a) Schematic model and (b) Equivalent circuit

In Fig. 2, $\alpha_{i}$ is the firing delay angle of converter $i, E_{d 0}$ is the maximum DC voltage of the batteries, $E_{b 1}$ is the battery overvoltage, $E_{b t}$ is the terminal of equivalent battery, $E_{b o c}$ is the battery open circuit voltage, $I_{B E S S}$ is the DC current though the battery, $P_{B E S S}$ is the active power provided by the batteries, $r_{b t}$ is the connecting resistance, $r_{b s}$ is the battery internal resistance, $r_{b p}$ is the self discharge resistance, $r_{b 1}$ is the overvoltage resistance, $\Delta f$ is the frequency deviation, $X_{c o}$ is the commutating reactance, $\Delta E_{c o}$ is the DC voltage without overlap, $K_{b}$ is the control loop gain, $T_{b}$ is the measurement device time constant.

The structure of BESS consists of power converters, battery cells and control parts [6] which are shown in Fig. 2 (a). From the schematic structure of BESS, the output of DC voltage is shown as:

$$
E_{d o}=\frac{6 \sqrt{6}}{\pi} E_{t}
$$

where $E_{t}$ is $\mathrm{AC}$ voltage between the line to neutral.

The equivalent circuit of BESS consists of a converter connected to an equivalent battery as shown in Fig. 2 (b). The terminal voltage of the equivalent battery can be calculated from:

$$
E_{b t}=E_{d o} \cos \alpha-I_{B E S S} R_{c}=\frac{3 \sqrt{6}}{\pi} E_{t}\left(\cos \alpha_{1}+\cos \alpha_{2}\right)-\frac{6}{\pi} I_{B E S S} X_{c o}
$$

According to the equivalent circuit of BESS, the expression of DC current flowing into the battery can be expressed as:

$$
I_{B E S S}=\frac{E_{b t}-E_{b o c}-E_{b 1}}{r_{b s}+r_{b t}}
$$

where

$$
\begin{aligned}
& E_{b o c}=\frac{r_{b p}}{1+S T_{b p}} I_{B E S S} \\
& E_{b 1}=\frac{r_{b 1}}{1+S T_{b 1}} I_{B E S S} \\
& T_{b p}=C_{b p} r_{b p} \\
& T_{b 1}=C_{b 1} r_{b 1}
\end{aligned}
$$

From the converter circuit analysis, the active and reactive power is absorbed by BESS as:

$$
\begin{gathered}
P_{B E S S}=\frac{3 \sqrt{6}}{\pi} I_{B E S S} E_{t}\left(\cos \alpha_{1}+\cos \alpha_{2}\right) \\
Q_{B E S S}=\frac{3 \sqrt{6}}{\pi} I_{B E S S} E_{t}\left(\sin \alpha_{1}+\sin \alpha_{2}\right)
\end{gathered}
$$

Only incremental active power is considered in load frequency control. Thus, $P$-modulation strategy is introduced to this paper. For $P$-modulation, $\alpha_{1}=-\alpha_{2}=\alpha$. Therefore; 


$$
\begin{aligned}
& P_{B E S S}=\frac{6 \sqrt{6}}{\pi} I_{B E S S} E_{t} \cos \alpha \\
& Q_{B E S S}=0 \\
& \Delta P_{B E S S}=I_{B E S S}^{0} \Delta E_{d}
\end{aligned}
$$

Then, the use of BESS in load frequency control is reached by a damping signal $\Delta E_{d .}$ :

$$
\Delta E_{d}=\frac{K_{b}}{1+S T_{b}} \Delta f
$$

The $\Delta f$ is the beneficial feedback from the power system in order to provide a damping effect. Linearizing equations (1) to (13), the block diagram of BESS can be shown in Fig. 3.

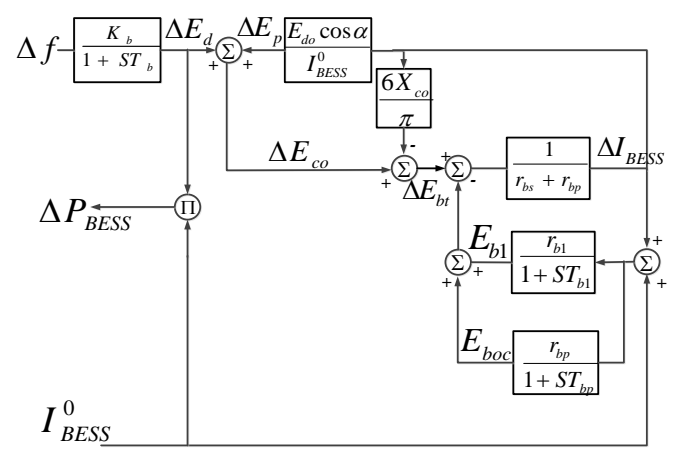

Fig. 3. Linearized BESS model for load frequency control

\section{Proposed Control Method}

\subsection{Structure of the developed program}

DIgSILENT PowerFactory is a computer aided engineering software for the analysis of industrial, utility and commercial electrical power system. This program provides the programming language called DIgSILENT Programming Language (DPL) used for developing automated scripts which connects other objects or elements. The structure of this language is similar to $C++$ language. Fig. 4 shows the structure of the DPL script used in developing the optimal sizing of BESS.

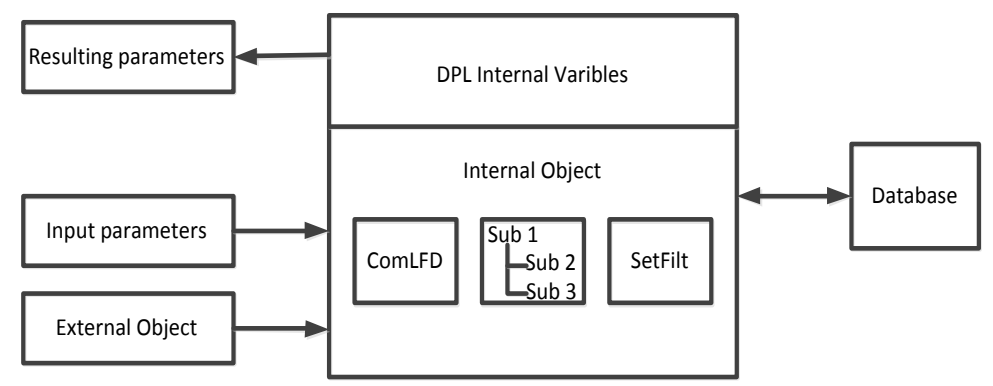

Fig. 4. Structure of DPL script for optimal sizing of BESS with load shedding scheme.

\subsection{Optimal sizing of BESS-based analytic algorithm with load shedding scheme}

If the generation cannot match the load demand in a short time during a large disturbance such as three phase fault, the system frequency will drop drastically. This significant drop will likely cause further extreme consequence such as system collapse. In this case, under frequency load shedding plays an important role in frequency control and BESS can improve the performance of frequency control in load shedding scheme. In this part, the optimal sizing of BESS with load shedding is accomplished by using 
DPL script in DIgSILENT PowerFactory, and the performance of the system frequency is discussed in the simulation results.

The overall flow chart of developed program for optimal sizing of BESS-based analytic algorithm with load shedding is shown in Fig. 5. The proposed analytic algorithm is implemented by using the DPL script with the structure in Fig. 4. The solution algorithm of this method is as follows:

Step 1) Read the input data of the microgrid system in DIgSILENT PowerFactory program and get the power values of photovoltaic $\left(P_{P V}\right)$, hydro generator $\left(P_{H V}\right)$, mini hydro generator $\left(P_{M i n i}\right)$ and the load 1 to $5\left(P_{L}\right)$.

Step 2) Set the desired power of load shedding $\left(P_{S}\right)$. Load shedding is based on the measured power imported by the grid at the moment of islanding.

Step 3) Calculate the total power of all loads $\left(P_{L T}\right)$ after setting the load shedding. Then, the program calculates the differential power $\left(P_{D P}\right)$ between generations and loads.

Step 4) Set the differential power $\left(P_{D P}\right)$ equal to the power of BESS $\left(P_{B}\right)$.

Step 5) Check the condition if the system frequency is within the values set by the user. If the system frequency does not satisfy the condition, the program will increase $P_{B E S S}$ by $0.01 \mathrm{MW}$ and the condition is run again. This step will continue until it meets the condition.

Step 6) End of process.

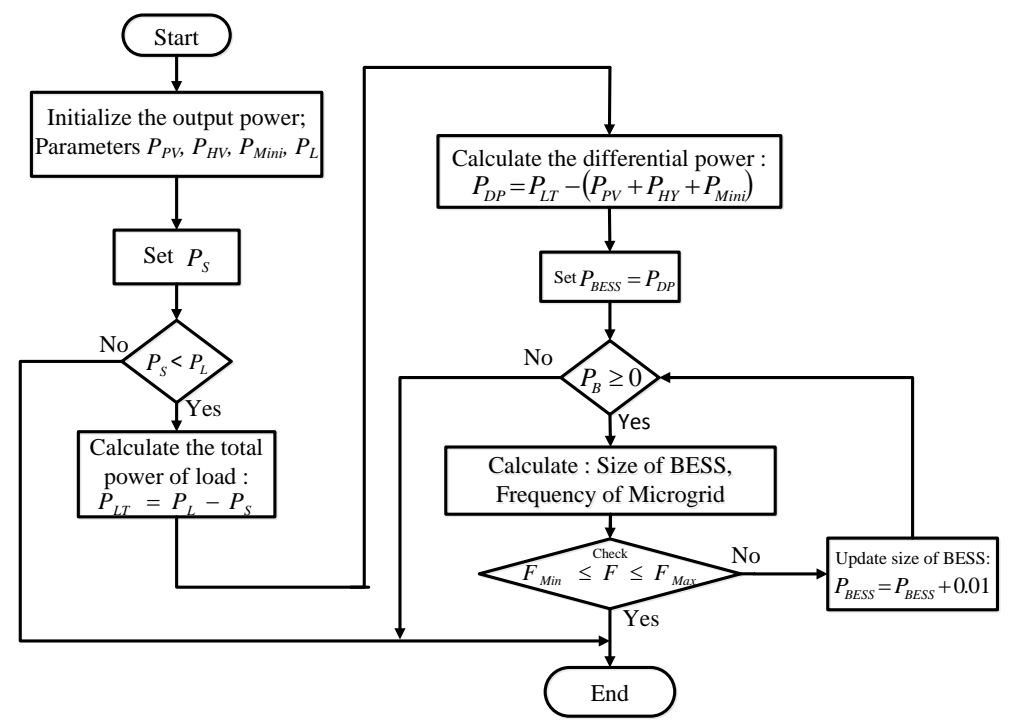

Fig. 5. Overall flow chart of developed program for optimal sizing of BESS-based analytic algorithm with load shedding scheme

\subsection{Objective function}

To minimize the power of BESS, the final objective function is chosen and expressed as:

$$
\text { Minimize } f=\min \left(P_{B E S S}\right)
$$

under the constraints of: 1) $F_{\min } \leq F \leq F_{\max }$ and 2) $P_{B E S S}^{\min } \leq P_{B E S S} \leq P_{B E S S}^{\max }$, where $F$ is the frequency of the microgrid system $(\mathrm{Hz})$ and $P_{B E S S}$ is the active power of BESS (MW).

\section{Simulation Results}

The proposed program is applied to the microgrid system under study as shown in Fig. 6. There are three power plants namely Mini-hydro, Hydro, and Photovoltaic with average output power of $1.2 \mathrm{MW}, 2$ MW and $3 \mathrm{MW}$ respectively. Moreover, there are two critical loads with power of $1.85 \mathrm{MW}$ and $1.9 \mathrm{MW}$ (Load 1, Load 4), and three non-critical loads with power of 1.7 MW, 1.75 MW and 2.4 MW (Load 2, 
Load 3, Load 5) at the moment of islanding. A three phase fault has occurred at bus HOA_1 at $\mathrm{t}=10.0 \mathrm{~s}$ and the system has applied the load shedding scheme to the non-critical load 5. Normally, the time to clear the fault and restore the system after the islanding operation occurred takes around 1 hour. Thus, this paper assumes that BESS can support the microgrid system for about 1 hour after the system is isolated from the main grid. This microgrid system is implemented in DIgSILENT PowerFactory Version 14.1.

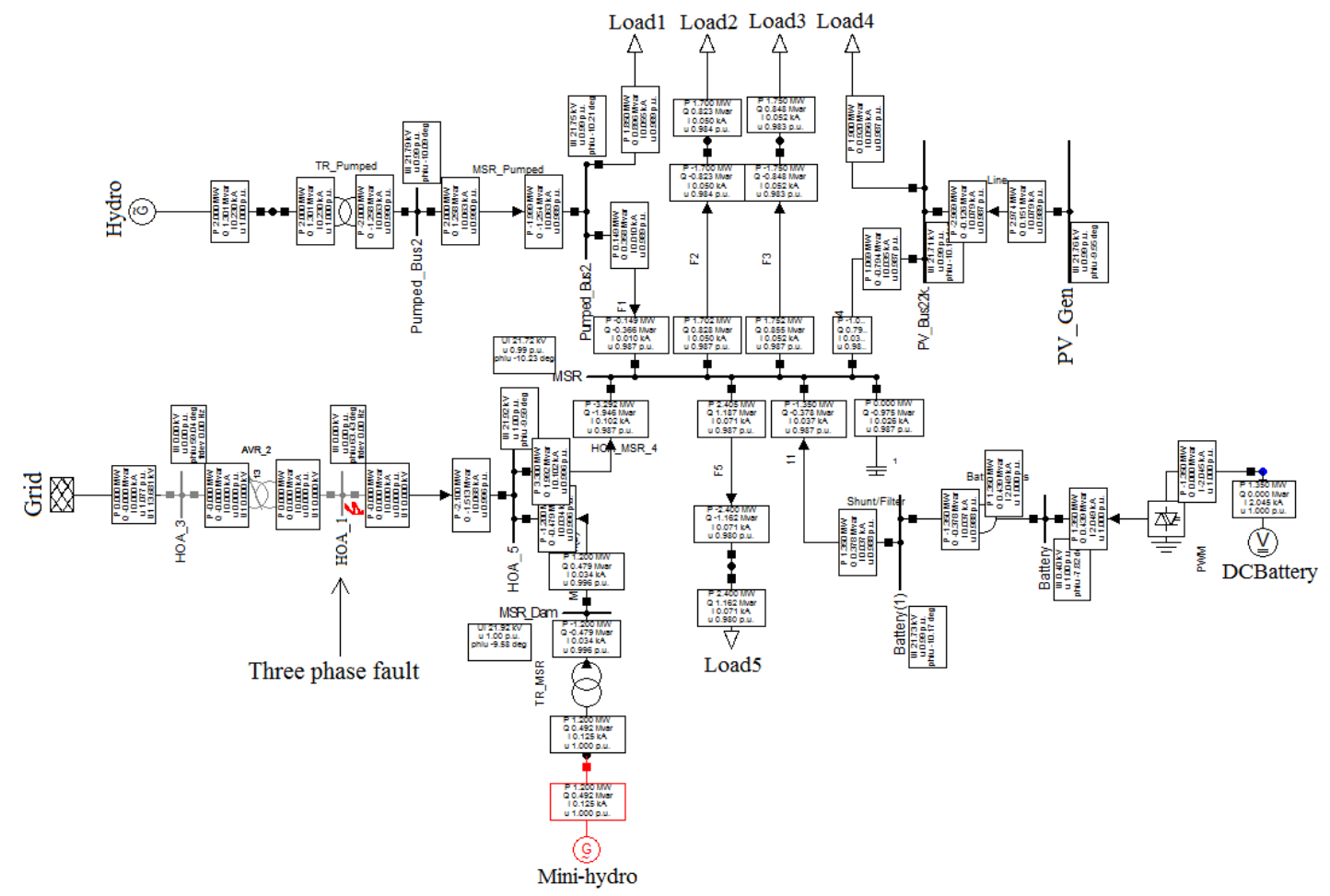

Fig. 6. Microgrid system is simulated using DIgSILENT PowerFactory

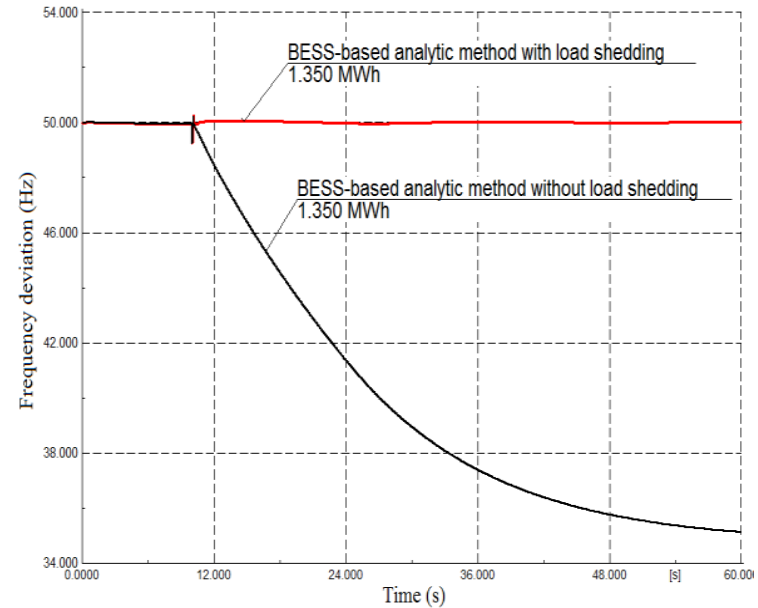

(a)

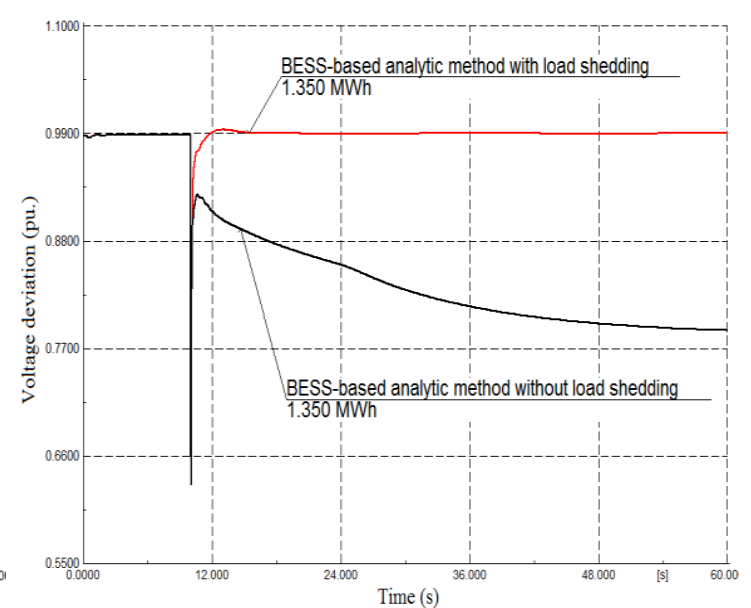

(b)

Fig. 7. System improvement with load shedding scheme: (a) frequency deviation and (b) voltage deviation.

In a case where the system cannot apply load shedding scheme when a three phase fault occurs at bus HOA_1, the system will collapse as shown in Fig. 7 (a) and (b).

Due to the three phase fault, load shedding is a method of frequency control protection. In this paper, BESS and load shedding scheme operate simultaneously. The performance of the system frequency with 
optimal size of BESS and load shedding scheme is observed and shown in Fig. 8(a). From this figure, the magnitude of the frequency deviation without the optimal size of BESS is higher and takes more time to stabilize than the optimal sizing of BESS-based analytic algorithm. In case of no BESS, the system frequency dropped drastically because the power supply cannot meet the load demand. It can be seen in Table 1 that in case of the optimal sizing of BESS, the performance of system frequency is much better when optimal sizing method of BESS is applied simultaneously with load shedding scheme. The influence of BESS is the essential point in this paper and $\mathrm{df} / \mathrm{dt}$ method is not included.

Table 1. Frequency and voltage of the microgrid system for each case after islanding operation

\begin{tabular}{cccc}
\hline \hline Case & $\begin{array}{c}\text { BESS } \\
(\mathrm{MW})\end{array}$ & $\begin{array}{c}\text { Frequency (Hz) } \\
\text { Reference : 50.0000 }\end{array}$ & $\begin{array}{c}\text { Voltage (pu.) } \\
\text { Reference : 0.9890 }\end{array}$ \\
\hline No BESS & 0 & 31.0258 & 0.8405 \\
\hline BESS without optimal size & 2.000 & 50.3011 & 0.9950 \\
\hline BESS-based analytic method & 1.350 & 50.1445 & 0.9897 \\
\hline \hline
\end{tabular}

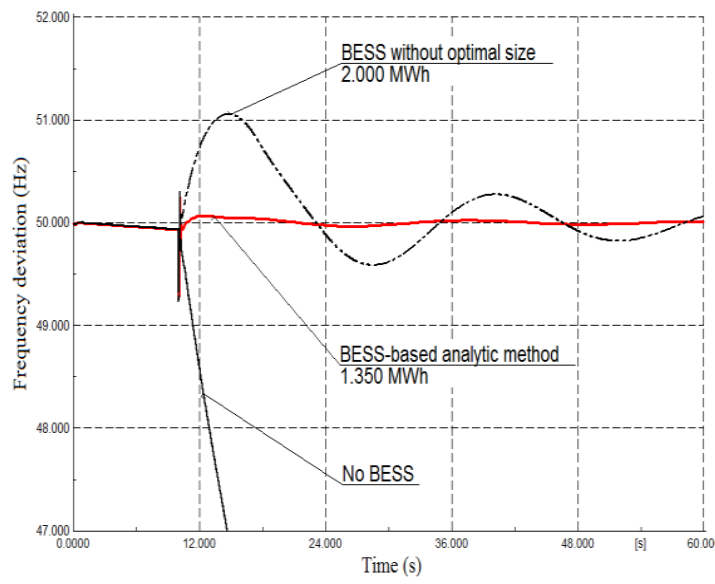

(a)

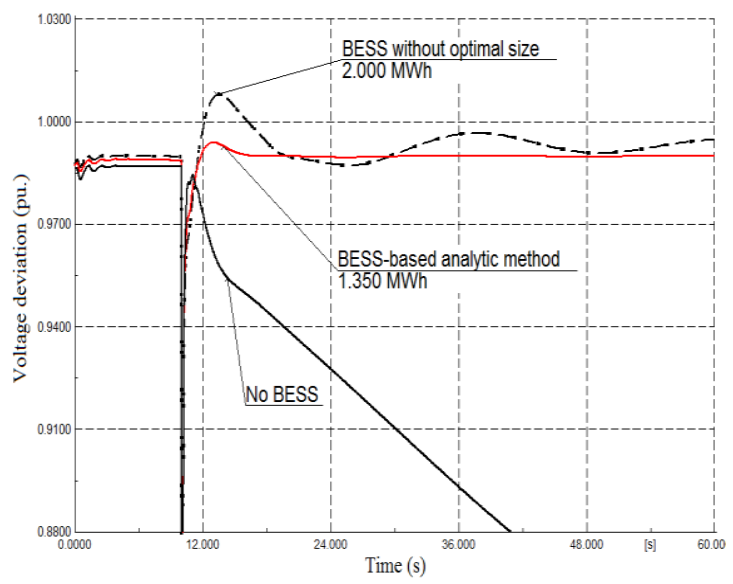

(b)

Fig. 8. Microgrid system after islanding operation: (a) Frequency deviation and (b) Voltage deviation

Moreover, the voltage deviation of the microgrid system is shown in Fig. 8(b). Based on this figure, the proposed method can maintain the voltage deviation better than the other cases. It is demonstrated that the optimal size of BESS-based analytic algorithm with load shedding scheme is more robust and stable than those without optimal size of BESS.

\section{Conclusions}

This paper proposes a control scheme using the optimal sizing of BESS-based analytic algorithm with load shedding scheme in order to improve the system frequency after islanding occurred, and to reduce the operating cost of microgrid. Based on the results, BESS has shown a good performance in load frequency control and can be used for emergency control purposes when the system load shedding is unavoidable. It is validated that the optimal size of BESS-analytic algorithm with load shedding scheme can give better stability than the case without optimal size of BESS. Moreover, the proposed method can also provide sustainable voltage to the system much better compared to other cases. In addition, the usage of optimal BESS with load shedding scheme was clearly illustrated and approved. For future work, to obtain a faster solution, this work will implement an intelligent technique to find the optimal size of BESS.

\section{References}

[1] Karimi H, Nikkhajoei H, Iravani R. Control of an electronically coupled distributed resource unit subsequent to an islanding event. IEEE Trans. Power Delivery, 2008; 23(1): 293-501. 
[2] Wai R, Wang W. Grid-connected photovoltaic generation system. IEEE. Trans. Circuits System, 2008; 55(3): 953-964.

[3] Katiraei F, Iravani M, Lehn P. Micro-grid autonomous operation during and subsequent to islanding process. IEEE Trans. Power Delivery, 2005; 20(1): 248-257.

[4] Mercier P, Cherkaoui R, Oudalov A. Optimizing a battery energy storage system for frequency control application in an isolated power system. IEEE Trans. Power System, 2009; 24: 1469-1477.

[5] Oudalov A, Chartouni D, Ohler C. Optimizing a battery energy storage system for primary frequency control. IEEE Trans. Power System, 2007; 22: 1259-1266.

[6] Halevi Y, Kottick D. Optimization of load shedding system. IEEE Trans. Energy Conversion, 1993; 8:207-213.

[7] Anderson P.M, Mirheydar M. A low-order frequency response model. IEEE Trans. Power System, 1990; 5:720-729.

[8] Anderson P.M, Mirheydar M. An adaptive method for setting underfrequency load shedding relays. IEEE Trans. Power System, 1992; 7:647-655.

[9] Kellogg W, Nehrir M, Venkataramanan G, Gerez V. Generation unit sizing and cost analysis for standard-alone wind, photovoltaic, and hybrid wind/PV systems. IEEE Trans. Energy Conversion, 1998; 13:70-75.

[10] Shrestha G, Goel L. A study on optimal sizing of stand-alone photovoltaic stations. IEEE Trans. Energy Conversion, 1998; 13:373-378. 